\title{
TWISTOR SECTIONS OF DIRAC BUNDLES
}

\author{
SERGIO A. H. CARDONA ${ }^{\dagger}$, PEDRO SOLÓRZANO*, AND IVÁN TÉLLEZ
}

\begin{abstract}
A Dirac bundle is a euclidean bundle over a riemannian manifold $M$ which is a compatible left $\mathrm{C} \ell(M)$-module, together with a metric connection also compatible with the Clifford action in a natural way. We prove some vanishing theorems and introduce the twistor equation within this framework. In particular, we exhibit a characterization of solutions for this equation in terms of the Dirac operator $D$ and a suitable Weitzenböcktype curvature operator $\mathcal{R}$. Finally, we analyze the especial case of the Clifford bundle to prove existence of nontrivial solutions of the twistor equation on spheres.
\end{abstract}

\section{INTRODUCTION}

The main purpose of this paper is to study some basic properties of Dirac bundles; in particular, the existence of vanishing theorems and of solutions to some classically motivated equations such as the twistor equation or the Killing equation. Dirac bundles are one possible generalization of spin bundles to the case when no spin structure is required. In the latter, the aforementioned equations have been extensively studied, see for example [4, 6 8, 10, 11, 14, 17]. As mentioned in the abstract, a Dirac bundle is a euclidean bundle over a riemannian manifold $M$ which is a compatible left $\mathrm{C} \ell(M)$-module, together with a metric connection also compatible with the Clifford action in a particular sense. With the above data a natural Dirac operator $D$ can be defined. The definition of a Dirac bundle was introduced in [15]. Therein, some of the properties of Dirac bundles are developed and classical vanishing theorems of Bochner and Lichnerowicz are proved using a generalized Bochner identity, where the corresponding curvature term $\mathcal{R}$ is constructed using the curvature of the Dirac bundle. We call $\mathcal{R}$ the Weitzenböck curvature operator of the Dirac bundle.

One of the fundamental ideas of twistor theory is that the geometry of a real structure, such as space-time, may be studied using an associated complex space [18]; regarding the geometry and properties of the real structure as derived from this complex space. This idea has been applied to reformulate various physical concepts in terms of twistors [20] and has been adapted and developed in mathematics by many authors. For example, Atiyah and Ward [1] used the Penrose twistor transform to give a correspondence between

2010 Mathematics Subject Classification. Primary 53C28; Secondary 53C27, 53C07.

Key words and phrases. Dirac bundles, euclidean bundles, Dirac operators, twistors, Killing sections.

$(\dagger)$ CONACYT-UNAM Research fellow.

(*) CONACYT-UNAM Research fellow.

(†) This author was supported by FORDECYT Grant No. 265667. 
algebraic bundles on the complex projective 3-space and minimum action solutions for $S U(2)$ Yang-Mills fields in the euclidean 4-space.

In [19], Penrose introduced the so-called twistor equation for certain 4-manifolds. In [2] Atiyah, Hitchin and Singer showed that if $X$ is a self-dual 4-manifold then the projective bundle of anti-self-dual spinors inherits the structure of a complex analytic 3-manifold using solutions to a suitable twistor equation.

The study of the space of twistor spinors - solutions to the twistor equation on a spin manifold - has been carried out since then. There are special solutions to this equation called Killing spinors. It has been proved [7] that if a spin manifold $M$ carries a Killing spinor then $M$ is a locally-irreducible Einstein space. In fact, the spinor bundle over the sphere is trivialized by Killing spinors [3]. Also, if $(M, g)$ is a compact connected riemannian spin manifold carrying a nontrivial twistor spinor there exists an Einstein metric $g^{*}$, conformally equivalent to $g$, such that the space of twistor spinors on $(M, g)$ coincides with the space of Killing spinors on $\left(M, g^{*}\right)$. For a compendium of results and properties of twistor spinors see [4].

We extend the notion of twistor spinor to Dirac bundles by studying the natural translation of the twistor equation to this setting. The main results of this paper are the following.

Theorem A. If the Weitzenböck curvature operator $\mathcal{R}$ of a Dirac bundle over a compact riemannian manifold is positive semi-definite, then every section $\sigma$ of the kernel of the Dirac operator $D$ is parallel and satisfies

$$
\langle\mathcal{R} \sigma, \sigma\rangle=0
$$

Furthermore, if $\mathcal{R}$ is also positive definite at some point, then $D$ has trivial kernel.

This is an elementary generalization of a Bochner-type vanishing theorem when no spin structure is assumed nor required.

Theorem B. Let $(S, \nabla,\langle\cdot, \cdot\rangle)$ be a Dirac bundle over a compact riemannian $n$-manifold $M$, and $D$ and $\mathcal{R}$ the aforementioned Dirac and Weitzenböck curvature operators, respectively. $A$ section $\sigma$ of $S$ is a twistor section if and only if

$$
D^{2} \sigma=\frac{n}{n-1} \mathcal{R} \sigma
$$

Moreover, in this case,

$$
\nabla_{X} D \sigma=\frac{n}{n-2}\left[\frac{1}{n-1} X \cdot \mathcal{R}-\frac{1}{2} \operatorname{Ric}_{X}\right] \sigma,
$$

where $\operatorname{Ric}_{X}: S \rightarrow S$ is the Ricci curvature operator on $S$.

This theorem is a generalization of a well-known result of Lichnerowicz (see Proposition 2 in [16] p. 335) in the context of spin bundles.

Theorem C. On a Dirac bundle $S$ over a compact riemannian $n$-manifold $M$ the following are equivalent:

(i) A section $\sigma$ of $S$ is a Killing section with constant $\lambda$.

(ii) A section $\sigma$ of $S$ satisfies $D \sigma=-n \lambda \sigma$ and $\mathcal{R} \sigma=\lambda^{2} n(n-1) \sigma$. 
We would like to emphasize that the direction (i) implies (ii) in Theorem $\mathrm{C}$ is well-known in the geometry of spin bundles (See [4], pp. 30-31). The converse in that setting-to the best of our knowledge - is not in the literature. Notice that in particular Theorem C implies the equivalence between (i) and (ii) in the classical case of spin bundles.

Theorem D. The twistor sections of $\mathrm{C} \ell\left(S^{n}\right), n \geq 2$, are given by

$$
\sigma=c_{1}+d f_{1}+d^{*}\left(* f_{2}\right)+* c_{2},
$$

with constants $c_{1}, c_{2}$ and functions $f_{1}, f_{2}: S^{n} \rightarrow \mathbb{R}$ such that $\Delta f_{i}=n f_{i}$.

This characterizes the solutions to the twistor equation in this setting, thus exhibiting the existence of nontrivial solutions different from those of the classical spin case.

Theorems A, B, C and D appear below as Theorems 4.1, 5.4, 5.10, and 6.4 respectively.

In the Appendix we analyze the Killing equation on Clifford bundles over surfaces. In light of Theorem A.1, this equation is much more restrictive than the twistor equation.

\section{Contents}

1. Introduction

2. Notations and conventions

3. Dirac Bundles

4. Vanishing results

5. Twistor sections

6. The Clifford bundle case

\section{Notations AND CONVEntions}

Throughout this communication some familiarity with the theory of Clifford algebras and spin bundles is expected. Classical references for these topics can be found within [4, 7, 15].

From now on, $M$ denotes an $n$-dimensional riemannian manifold, $\mathrm{C} \ell(M)$ its Clifford bundle, and it is assumed that all associated bundles (tangent, cotangent, exterior, Clifford, etc.) are endowed with the corresponding naturally induced metrics $\langle\cdot, \cdot\rangle$ and metric connections $\nabla$. The corresponding pointwise norms is denoted by $|\cdot|$.

We also adhere to the following conventions. Tangent vectors and vector fields are denoted with Latin capital letters $X, Y$, etc. and the space of vector fields by $\mathfrak{X}(M)$. Bundles over $M$ are denoted by $E, S$, etc., and their spaces of sections (resp. compactlysupported sections) are denoted by $\Gamma(E)$, etc. (resp. by $\Gamma_{\mathrm{c}}(E)$, etc.). Individual elements and sections are denoted by Greek letters $\sigma, \tau$ etc., except for elements and sections of the Clifford bundle $\mathrm{C} \ell(M)$, which — within the context of Clifford multiplication - are denoted by Latin letters $a, b$, etc. The fiber over a point $p$ in $M$ of a bundle $E$ is denoted by $E_{p}$. Likewise, to avoid possible ambiguities, elements of $E_{p}$ are sometimes denoted as $\sigma_{p}$. 


\section{Dirac Bundles}

In this section we review the notion of Dirac bundle introduced in [15], mention some fundamental properties and prove some basic new ones. A Dirac bundle is a generalization a spin bundle to the case when no spin structure is required. Hence, it is possible to define a Dirac operator on such bundles. We also recall the definitions of the Weitzenböck curvature operator - prove some elementary properties of it - and of the connection laplacian of a Dirac bundle, and review the generalized Bochner identity that relates them.

Definition 3.1 (Dirac bundle). A bundle $S$ over $M$ is a Dirac bundle if it is a left $\mathrm{C} \ell(M)-$ module, together with a euclidean metric $\langle\cdot, \cdot\rangle$ and a metric connection $\nabla$, satisfying the following two conditions:

(i) For each $X \in T_{p} M$ and $\sigma, \tau \in S_{p}$

$$
\langle X \cdot \sigma, \tau\rangle+\langle\sigma, X \cdot \tau\rangle=0
$$

(ii) for all $a \in \Gamma(\mathrm{C} \ell(M))$ and $\sigma \in \Gamma(S)$,

$$
\nabla(a \cdot \sigma)=(\nabla a) \cdot \sigma+a \cdot \nabla \sigma .
$$

Spin bundles are automatically Dirac bundles. Another immediate example of a Dirac bundle is the Clifford bundle of $M$ itself. Since as a vector bundle $\mathrm{C} \ell(M)$ is isomorphic to the exterior bundle (see [15] p. 10), the existence of Dirac bundles is not topologically obstructed as in the case of the bundle of spinors on a spin manifold. Several of the algebraic properties of the Clifford action that are well known in the spin case also hold here, e.g. from (ii) it follows that for $X \in T_{p} M$ and $\sigma \in S_{p}$

$$
|X \cdot \sigma|=|X||\sigma| \text {. }
$$

Another property of $\mathrm{C} \ell(M)$ that is required in the sequel is the following (see [7] p. 6. for details): there exists a fiberwise-linear involution $\gamma$ on $\mathrm{C} \ell(M)$ such that for all $X \in T_{p} M$

$$
\gamma_{p}(X)=X
$$

and for all $a, b \in \mathrm{C} \ell(M)_{p}$

$$
\gamma_{p}(a \cdot b)=\gamma_{p}(b) \cdot \gamma_{p}(a)
$$

Notice that from (3.5) it is readily verified that for any $a \in \Gamma(\mathrm{C} \ell(M))$,

$$
\nabla(\gamma(a))=\gamma(\nabla a)
$$

where $\nabla$ is the natural connection on $\mathrm{C} \ell(M)$.

Recall that a parallel subbundle of a euclidean bundle with connection is a subbundle that is invariant under parallel translations; equivalently, if for any section of the subbundle its covariant derivative is also a section of the subbundle (cf. [13] p. 114).

The following proposition establishes some elementary properties of Dirac bundles.

Proposition 3.2. Let $S, S_{1}$ and $S_{2}$ be Dirac bundles and $E$ a euclidean vector bundle over $M$. The following are Dirac bundles over $M$ :

(i) Any parallel subbundle of $S$ which is also a left $\mathrm{C} \ell(M)$-submodule of $S$ with the restricted action from $S$. 
(ii) The Whitney sum $S_{1} \oplus S_{2}$ of $S_{1}$ and $S_{2}$ with the action defined componentwise.

(iii) The dual $S^{*}$ of $S$ with the action given by

$$
\left(a \cdot \sigma^{*}\right)(\xi)=\sigma^{*}(\gamma(a) \cdot \xi)
$$

for any $\sigma^{*} \in S^{*}$ and $a \in \mathrm{C} \ell(M)$ over the same point and $\gamma$ the aforementioned involution.

(iv) The tensor product $S \otimes E$ of $S$ and $E$ with the action given by

$$
a \cdot(\sigma \otimes \xi)=(a \cdot \sigma) \otimes \xi,
$$

for any $\sigma \in S, \xi \in E$ and $a \in \mathrm{C} \ell(M)$ over the same point.

Remark 3.3. A Dirac subbundle of a Dirac bundle is any subbundle that satisfies the conditions in (ii). Also, a Dirac bundle is irreducible if it has no nontrivial Dirac subbundles.

Remark 3.4. If $E$ is itself a Dirac bundle, then $S \otimes E$ can be considered as a Dirac bundle in two distinct ways. This is the case, e.g., for the bundle $\operatorname{Hom}(S, S)$ of a Dirac bundle $S$.

Proof of Proposition 3.2. The proof of (ii) and (iii) is straightforward and (iv) is proved in [15] pp. 121-122 as Proposition 5.10 therein. To establish (iii), observe that it is straightforward to verify that the action given by (3.7) induces a $\mathrm{C} \ell(M)$-module structure on $S^{*}$. Since $S$ is Dirac, (3.4), (3.7), and the definition of the metric dual yield that

$$
X \cdot \sigma^{*}=-(X \cdot \sigma)^{*}
$$

for $X \in T_{p} M$ and $\sigma \in S_{p}$. Now, (3.9) gives

$$
\begin{aligned}
\left\langle X \cdot \sigma^{*}, \tau^{*}\right\rangle & =-\left\langle(X \cdot \sigma)^{*}, \tau^{*}\right\rangle=-\langle X \cdot \sigma, \tau\rangle \\
& =\langle\sigma, X \cdot \tau\rangle=\left\langle\sigma^{*},(X \cdot \tau)^{*}\right\rangle=-\left\langle\sigma^{*}, X \cdot \tau^{*}\right\rangle
\end{aligned}
$$

establishing (ii) in Definition 3.1 for $S^{*}$.

On the other hand, the standard dual connection is given by

$$
\left(\nabla_{X} \sigma^{*}\right)(\tau)=X\left(\sigma^{*}(\tau)\right)-\sigma^{*}\left(\nabla_{X} \tau\right)
$$

with $X \in T_{p} M, \tau \in \Gamma(S)$ and $\sigma^{*} \in \Gamma\left(S^{*}\right)$. It follows from (3.7), (3.10) and (3.6) that

$$
\begin{aligned}
\left(\nabla_{X}\left(a \cdot \sigma^{*}\right)\right)(\tau)= & X\left(\left(a \cdot \sigma^{*}\right)(\tau)\right)-\left(a \cdot \sigma^{*}\right)\left(\nabla_{X} \tau\right) \\
= & X\left(\sigma^{*}(\gamma(a) \cdot \tau)\right)-\sigma^{*}\left(\gamma(a) \cdot \nabla_{X} \tau\right) \\
= & X\left(\sigma^{*}(\gamma(a) \cdot \tau)\right)+\sigma^{*}\left(\gamma\left(\nabla_{X} a\right) \cdot \tau\right) \\
& -\sigma^{*}\left(\gamma(a) \cdot \nabla_{X} \sigma\right)-\sigma^{*}\left(\nabla_{X}(\gamma(a)) \cdot \tau\right) \\
= & \sigma^{*}\left(\gamma\left(\nabla_{X} a\right) \cdot \tau\right)+X\left(\sigma^{*}(\gamma(a) \cdot \tau)\right)-\sigma^{*}\left(\nabla_{X}(\gamma(a) \cdot \tau)\right) \\
= & \left.\sigma^{*}\left(\gamma\left(\nabla_{X} a\right) \cdot \tau\right)+\left(\nabla_{X} \sigma^{*}\right)(\gamma(a) \cdot \tau)\right) \\
= & \left(\left(\nabla_{X} a\right) \cdot \sigma^{*}+a \cdot \nabla_{X} \sigma^{*}\right)(\tau),
\end{aligned}
$$

establishing (iii) in Definition 3.1 for $S^{*}$.

Lemma 3.5. The orthogonal complement of a Dirac subbundle is also a Dirac subbundle. 
Proof. Let $E \subset S$ be a Dirac subbundle of $S$. The proof that $E^{\perp}$ is also parallel is straightforward. To see that it is a $\mathrm{C} \ell(M)$-module as well, let $e_{1}, \ldots, e_{n}$ be a local orthonormal frame. By part (ii) of Definition 3.1,

$$
\left\langle e_{i_{1}} \cdot \ldots \cdot e_{i_{k}} \cdot \sigma, \tau\right\rangle=(-1)^{k}\left\langle\sigma, \gamma\left(e_{i_{1}} \cdot \ldots \cdot e_{i_{k}}\right) \cdot \tau\right\rangle
$$

which implies that for any $a \in \mathrm{C} \ell(M), \sigma, \tau \in S$, there exists $b \in \mathrm{C} \ell(M)$ such that

$$
\langle a \cdot \sigma, \tau\rangle=\langle\sigma, b \cdot \tau\rangle \text {. }
$$

Letting $\sigma \in E^{\perp}$ and $\tau \in E$, shows that $a \cdot \sigma \in E^{\perp}$.

Proposition 3.6. Dirac bundles of finite rank are the sum of irreducible Dirac bundles.

Proof. The proof is by induction on the rank of $S$ : Let $E$ be a nontrivial irreducible Dirac subbundle of $S$, then by Lemma 3.5, $E^{\perp}$ is a Dirac subbundle whose rank is strictly less than the rank of $S$.

On a Dirac bundle, it is possible to define a Dirac operator imitating the classical definition of the Dirac operator of a spin bundle. Consequently, the Dirac operator $D: \Gamma(S) \rightarrow$ $\Gamma(S)$ of a Dirac bundle is locally given by

$$
D \sigma=\sum_{i=1}^{n} e_{i} \cdot \nabla_{e_{i}} \sigma,
$$

where $e_{1}, \ldots, e_{n}$ is a local orthonormal frame of $M$. It is known that $D$ and $D^{2}$ are elliptic operators. Observe that $D$ satisfies the following Leibniz rule:

$$
D(f \sigma)=\nabla f \cdot \sigma+f D \sigma,
$$

for $f$ and $\sigma$ smooth. The formula

$$
(\sigma, \tau)=\int_{M}\langle\sigma, \tau\rangle
$$

defines an inner product 1 whose norm is denoted by $\|\cdot\|$, on the corresponding $L^{2}$-space. There, the Dirac operator $D$ is formally self-adjoint with respect to it, i.e.,

$$
(D \sigma, \tau)=(\sigma, D \tau)
$$

In particular, if $M$ is compact, $\operatorname{ker} D=\operatorname{ker}\left(D^{2}\right)$. An extension of this to complete riemannian manifolds is also known (see Theorem 5.7 in [15] p. 117). Here we present a different proof that is a consequence of the following result.

Proposition 3.7. Let $M$ be complete and let $S$ be a Dirac bundle over it. Then, for each $\sigma \in \Gamma(S)$ and $t>0$,

$$
\|D \sigma\|^{2} \leq t\left\|D^{2} \sigma\right\|^{2}+\frac{1}{t}\|\sigma\|^{2}
$$

\footnotetext{
$1_{\text {This }}$ and some subsequent constructions can be done on a euclidean vector bundle with a metric connection. Since the main purpose of this communication is to focus on Dirac bundles, we restrict our attention to them.
} 
Proof. (The arguments below follow closely those in [7] pp. 96-98.) Observe that there is nothing to prove if $\|\sigma\|$ is infinite; therefore assume that $\|\sigma\|$ is finite.

Since $M$ is complete, in particular it is metrically complete with respect to the shortest path metric $d$ and the metric balls,

$$
B_{p}(r)=\{q \in M: d(p, q)<r\}
$$

for each $p \in M$ and $r>0$, are pre-compact.

Fix a point $p_{0} \in M$ and let $B(r)$ denote $B_{p_{0}}(r)$. Using a standard argument of bump functions it is possible to define, for any $r>0$, a smooth function $f_{r}: M \rightarrow[0,1]$ such that $f_{r} \equiv 1$ on $B(r), \operatorname{supp} f_{r} \subset B(2 r)$, and

$$
\left|\nabla\left(f_{r}\right)\right|^{2} \leq \frac{C^{2}}{r^{2}}
$$

for a certain constant $C$ independent of $r$ (see [7] pp. 95-96 for details on this construction). For any $\varepsilon>0$ and $\sigma \in \Gamma(S)$, we have

$$
\begin{aligned}
\int_{B(2 r+\varepsilon)}\left|f_{r} D \sigma\right|^{2} & =\int_{B(2 r+\varepsilon)}\left\langle D\left(f_{r}^{2} D \sigma\right), \sigma\right\rangle \\
& =\int_{B(2 r+\varepsilon)}\left\langle 2 f_{r} \nabla\left(f_{r}\right) \cdot D \sigma, \sigma\right\rangle+\int_{B(2 r+\varepsilon)}\left\langle f_{r}^{2} D^{2} \sigma, \sigma\right\rangle .
\end{aligned}
$$

To show this, observe that in the first line, integration over $B(2 r+\varepsilon)$ coincides with integration over the whole $M$, and thus we can use (3.14). Using (i) of Definition 3.1 in the above expression and letting $\varepsilon$ go to zero yields

$$
\int_{B(2 r)}\left|f_{r} D \sigma\right|^{2}=\int_{B(2 r)}\left\langle D^{2} \sigma, f_{r}^{2} \sigma\right\rangle-\int_{B(2 r)}\left\langle f_{r} D \sigma, 2 \nabla\left(f_{r}\right) \cdot \sigma\right\rangle .
$$

The fact that, for any $t>0$,

$$
|\langle x, y\rangle| \leq \frac{t}{2}|x|^{2}+\frac{1}{2 t}|y|^{2}
$$

allows us to estimate each of the right-hand-side terms of (3.17). Indeed, from the definition of $f_{r}$, it yields

$$
\left|\int_{B(2 r)}\left\langle D^{2} \sigma, f_{r}^{2} \sigma\right\rangle\right| \leq \frac{t}{2} \int_{B(2 r)}\left|D^{2} \sigma\right|^{2}+\frac{1}{2 t} \int_{B(2 r)}|\sigma|^{2} .
$$

Also, for $t=1$, using (3.3) and (3.16),

$$
\left|\int_{B(2 r)}\left\langle f_{r} D \sigma, 2 \nabla\left(f_{r}\right) \cdot \sigma\right\rangle\right| \leq \frac{1}{2} \int_{B(2 r)}\left|f_{r} D \sigma\right|^{2}+\frac{2 C^{2}}{r^{2}} \int_{B(2 r)}|\sigma|^{2} .
$$

Applying the triangle inequality to (3.17), together with (3.18) and (3.19) - and grouping like terms - yields

$$
\int_{B(2 r)}\left|f_{r} D \sigma\right|^{2} \leq t \int_{B(2 r)}\left|D^{2} \sigma\right|^{2}+\left(\frac{1}{t}+\frac{4 C^{2}}{r^{2}}\right) \int_{B(2 r)}|\sigma|^{2} .
$$


Finally, since

$$
\int_{B(r)}|D \sigma|^{2} \leq \int_{B(2 r)}\left|f_{r} D \sigma\right|^{2}
$$

letting $r$ go to infinity in the previous two inequalities finishes the proof.

Corollary 3.8. Let $M$ be complete. In the space of $L^{2}$-section over $S$,

$$
\operatorname{ker} D=\operatorname{ker}\left(D^{2}\right)
$$

Proof. One inclusion is evident. Assume $D^{2} \sigma=0$ for an $L^{2}$-section $\sigma$ of $S$. Elementary elliptic theory yields that $\sigma \in \Gamma(S)$ (see [15] pp. 113 and 193 for details). From Proposition 3.7,

$$
\|D \sigma\|^{2} \leq \frac{1}{t}\|\sigma\|^{2}
$$

for any $t>0$. Therefore $D \sigma=0$.

The connection laplacian $\nabla^{*} \nabla: \Gamma(S) \rightarrow \Gamma(S)$ is defined by

$$
\nabla^{*} \nabla \sigma=-\operatorname{tr}\left(\nabla^{2} \sigma\right)
$$

for any $\sigma \in \Gamma(S)$, where for each $X, Y \in \mathfrak{X}(M)$ the operator $\nabla_{X, Y}^{2}: \Gamma(S) \rightarrow \Gamma(S)$ is given by $\nabla_{X, Y}^{2} \sigma=\nabla_{X} \nabla_{Y} \sigma-\nabla_{\nabla_{X} Y} \sigma$ (notice that it is tensorial in $X$ and $Y$ ). The name laplacian is justified since it satisfies

$$
\left(\nabla^{*} \nabla \sigma, \tau\right)=(\nabla \sigma, \nabla \tau)
$$

for all $\sigma, \tau \in \Gamma_{\mathrm{c}}(S)$; in the right-hand side, $\nabla \sigma$ and $\nabla \tau$ are regarded as sections of $T^{*} M \otimes S$. In terms of a local orthonormal frame $e_{1}, \ldots, e_{n}$,

$$
\nabla^{*} \nabla \sigma=-\sum_{j=1}^{n} \nabla_{e_{j}, e_{j}}^{2} \sigma
$$

and

$$
(\nabla \sigma, \nabla \tau)=\sum_{j}\left(\nabla_{e_{j}} \sigma, \nabla_{e_{j}} \tau\right)
$$

From (3.21) it is evident that the connection laplacian is nonnegative and formally selfadjoint. Moreover, in the compact case (3.21) implies that $\nabla^{*} \nabla \sigma=0$ if and only if $\sigma$ is parallel (details in [15] pp. 154-155).

The Weitzenböck curvature operator of a Dirac bundle $S$ over $M$ is the linear operator $\mathcal{R}: S \rightarrow S$ defined for a local orthonormal frame $e_{1}, \ldots, e_{n}$ by the formula

$$
\mathcal{R} \sigma=\sum_{i<j} e_{i} \cdot e_{j} \cdot R\left(e_{i}, e_{j}\right) \sigma
$$

This operator is well-defined and satisfies the generalized Bochner identity (see [15], p. $155)$

$$
D^{2}=\nabla^{*} \nabla+\mathcal{R}
$$


Notice that it is evident from the Bochner identity that $\mathcal{R}$ is formally self-adjoint, since both $D$ and $\nabla^{*} \nabla$ are too (cf. (3.14) and (3.21) ). For $\mathcal{R}$ even more is true, as the following proposition shows.

Proposition 3.9. The Weitzenböck operator of a Dirac bundle is pointwise self-adjoint.

Proof. Let $e_{1}, \ldots, e_{n}$ be a local orthonormal frame. It is easy to verify that

$$
R\left(e_{i}, e_{j}\right)\left(e_{k} \cdot \sigma\right)=e_{k} \cdot R\left(e_{i}, e_{j}\right) \sigma
$$

Using this and the standard fact that $\left\langle R\left(e_{i}, e_{j}\right) \sigma, \tau\right\rangle=-\left\langle\sigma, R\left(e_{i}, e_{j}\right) \tau\right\rangle$,

$$
\begin{aligned}
\langle\mathcal{R} \sigma, \tau\rangle & =\sum_{i<j}\left\langle e_{i} \cdot e_{j} \cdot R\left(e_{i}, e_{j}\right) \sigma, \tau\right\rangle \\
& =\sum_{i<j}\left\langle\sigma, R\left(e_{i}, e_{j}\right)\left(e_{i} \cdot e_{j} \cdot \tau\right)\right\rangle \\
& =\sum_{i<j}\left\langle\sigma, e_{i} \cdot e_{j} \cdot R\left(e_{i}, e_{j}\right) \tau\right\rangle \\
& =\langle\sigma, \mathcal{R} \tau\rangle .
\end{aligned}
$$

The next proposition provides specific formulas for the Weitzenböck operator of the bundles described in Proposition 3.2.

Proposition 3.10. Let $\mathcal{R}$ denote the Weitzenböck operator on each of the following cases.

(i) On a Dirac subbundle $\mathcal{R}$ is the restriction of the ambient Weitzenböck operator.

(ii) On the Whitney sum of two Dirac bundles,

$$
\mathcal{R}(\xi, \zeta)=(\mathcal{R} \xi, \mathcal{R} \zeta)
$$

(iii) On the dual of a Dirac bundle,

$$
\mathcal{R}\left(\sigma^{*}\right)=(\mathcal{R} \sigma)^{*} .
$$

(iv) On a tensor product of a Dirac bundle and a euclidean bundle, locally,

$$
\mathcal{R}(\sigma \otimes \xi)=\mathcal{R} \sigma \otimes \xi+\sum_{i<j} e_{i} \cdot e_{j} \cdot \sigma \otimes R\left(e_{i}, e_{j}\right) \xi
$$

where $e_{1}, \ldots, e_{n}$ is a local orthonormal frame.

Remark. The last term of the right-hand side of (3.28) is already defined in [15] p. 164. It could be described invariantly as $\operatorname{tr}(\omega \mapsto \omega \cdot \sigma \otimes R(\omega) \xi)$, with $\omega \in \Lambda^{2} T M$. Proposition 4.6 below uses a real-valued version of this trace.

Proof of Proposition 3.10. Parts (i) and (ii) are immediate. To see parts (iii) and (iv), let $e_{1}, \ldots, e_{n}$ be a local orthonormal frame. For the dual bundle, let $\sigma, \eta \in S$ over the same 
point. Then, since $R(X, Y)\left(\sigma^{*}\right)=(R(X, Y) \sigma)^{*}$,

$$
\begin{aligned}
{\left[\mathcal{R}\left(\sigma^{*}\right)\right](\eta) } & =\sum_{i<j} e_{i} \cdot e_{j} \cdot R\left(e_{i}, e_{j}\right)\left(\sigma^{*}\right)(\eta)=\sum_{i<j} e_{i} \cdot e_{j} \cdot\left(R\left(e_{i}, e_{j}\right) \sigma\right)^{*}(\eta) \\
& =\sum_{i<j}\left\langle R\left(e_{i}, e_{j}\right) \sigma, \gamma\left(e_{j}\right) \cdot \gamma\left(e_{i}\right) \cdot \eta\right\rangle=\sum_{i<j}\left\langle e_{i} \cdot e_{j} \cdot R\left(e_{i}, e_{j}\right) \sigma, \eta\right\rangle \\
& =\langle\mathcal{R} \sigma, \eta\rangle=(\mathcal{R} \sigma)^{*}(\eta) .
\end{aligned}
$$

For the tensor product, let $\sigma, \xi \in S$ over the same point. Then

$$
\begin{aligned}
\mathcal{R}(\sigma \otimes \xi) & =\sum_{i<j} e_{i} \cdot e_{j} \cdot R\left(e_{i}, e_{j}\right)(\sigma \otimes \xi) \\
& =\sum_{i<j} e_{i} \cdot e_{j} \cdot\left(R\left(e_{i}, e_{j}\right) \sigma \otimes \xi+\sigma \otimes R\left(e_{i}, e_{j}\right) \xi\right) \\
& =\mathcal{R} \sigma \otimes \xi+\sum_{i<j} e_{i} \cdot e_{j} \cdot \sigma \otimes R\left(e_{i}, e_{j}\right) \xi .
\end{aligned}
$$

\section{VANishing RESUlts}

The purpose of this section is to prove Theorem A as well as to establish some basic properties related to the positivity of the Weitzenböck operator. As a general principle, Bochner-type identities gives rise to Bochner vanishing theorems, e.g. in Complex Geometry there is a Bochner identity relating the Chern connection and the mean curvature for any holomorphic vector bundle. Such a result together with some conditions on the mean curvature implies the parallelism or nonexistence of holomorphic sections (see [12] ch. 3). In our context this is also the case: the Bochner identity (3.25) together with a positivity condition implies Theorem A, which is a particular type of Bochner vanishing theorem.

Theorem 4.1. If the Weitzenböck curvature operator $\mathcal{R}$ of a Dirac bundle over a compact manifold is positive semi-definite, then every section $\sigma$ of the kernel of the Dirac operator $D$ is parallel and satisfies

$$
\langle\mathcal{R} \sigma, \sigma\rangle=0
$$

Furthermore, if $\mathcal{R}$ is positive definite at some point, then $D$ has trivial kernel.

Proof. To see the first claim, assume $\mathcal{R}$ is positive semi-definite and let $\sigma$ be a section in the kernel of $D$. Then $\langle\mathcal{R} \sigma, \sigma\rangle \geq 0$ and by (3.21) and (3.25),

$$
0=\left(D^{2} \sigma, \sigma\right)=\left(\nabla^{*} \nabla \sigma, \sigma\right)+(\mathcal{R} \sigma, \sigma)=\|\nabla \sigma\|^{2}+(\mathcal{R} \sigma, \sigma) .
$$

Being nonnegative, the two terms on the right-hand side of the last equation must vanish, therefore $\sigma$ is parallel and satisfies (4.1).

Furthermore, assume $\mathcal{R}$ is also positive definite at a point. Therefore it is positive definite in a neighborhood and $(\mathcal{R} \sigma, \sigma)>0$ for any $\sigma$ that doesn't vanish identically on that neighborhood. Now let $\sigma$ be a section in the kernel of $D$. By the first part it is parallel

\footnotetext{
${ }^{2}$ Recall that a self-adjoint operator $\mathcal{P}$ is positive semi-definite (resp. definite) if for all $v$ (resp. for $v \neq 0$ ) in its domain $\langle\mathcal{P} v, v\rangle \geq 0$ (resp. $\langle\mathcal{P} v, v\rangle>0)$.
} 
and $(\mathcal{R} \sigma, \sigma)=0$. Being parallel, if $\sigma$ is nonzero at a point, it is nonzero everywhere. Thus, $\sigma$ is identically zero.

Next we study some further properties of the Weitzenböck operator and establish conditions under which its positivity is preserved under the bundle constructions considered in Proposition 3.2. The Weitzenböck operator on any Dirac subbundle inherits any positivity from the ambient. For the Whitney sum and the dual we have the following results.

Proposition 4.2. The Weitzenböck operator on the Whitney sum of Dirac bundles is positive semi-definite (resp. definite) if and only if the Weitzenböck operator on each summand is positive semi-definite (resp. definite).

Proof. Both directions follow readily from (3.26).

Proposition 4.3. The Weitzenböck operator on a Dirac bundle is positive semi-definite (resp. definite) if and only if the Weitzenböck operator on its dual is positive semi-definite (resp. definite).

Proof. Both directions follow readily from (3.27).

The case of the tensor product requires a more detailed analysis.

Lemma 4.4. Let $S$ and $E$ be euclidean bundles over $M$ and assume further that $S$ is Dirac. The Weitzenböck operator on $S \otimes E$ satisfies that

$$
\langle\mathcal{R}(\sigma \otimes \xi), \sigma \otimes \xi\rangle=\langle\mathcal{R} \sigma, \sigma\rangle\langle\xi, \xi\rangle
$$

for any $\sigma \in S$ and $\xi \in E$ over the same point.

Proof. From (3.28) it follows that

$$
\begin{aligned}
\langle\mathcal{R}(\sigma \otimes \xi), \tau \otimes \zeta\rangle & =\langle\mathcal{R} \sigma \otimes \xi, \tau \otimes \zeta\rangle+\sum_{i<j}\left\langle e_{i} \cdot e_{j} \cdot \sigma \otimes R\left(e_{i}, e_{j}\right) \xi, \tau \otimes \zeta\right\rangle \\
& =\langle\mathcal{R} \sigma, \tau\rangle\langle\xi, \zeta\rangle+\sum_{i<j}\left\langle e_{i} \cdot e_{j} \cdot \sigma, \tau\right\rangle\left\langle R\left(e_{i}, e_{j}\right) \xi, \zeta\right\rangle .
\end{aligned}
$$

Taking $\tau \otimes \zeta=\sigma \otimes \xi$ finishes the proof.

Proposition 4.5. Let $S$ and $E$ be euclidean bundles over $M$ and assume further that $S$ is Dirac. The Weitzenböck operator on $S$ is positive semi-definite (resp. definite) if the Weitzenböck operator on $S \otimes E$ is also positive semi-definite (resp. definite).

Proof. If the Weitzenböck operator on $S \otimes E$ is positive semi-definite then $\langle\mathcal{R} \eta, \eta\rangle \geq 0$ (resp. $\langle\mathcal{R} \eta, \eta\rangle>0$ ) for any $\eta \in S \otimes E$ (resp. for $\eta \neq 0$ ). In particular, by letting $\eta=\sigma \otimes \xi$ the result now follows from (4.2).

It is important to mention that in other contexts these types of definiteness are inherited in tensor products (see for instance [12] p. 53); to accomplish that, both factors must possess such property. For Dirac bundles, even though the Dirac structure on $S \otimes E$ is defined only using the Dirac structure of $S$, (4.3) shows that the Weitzenböck operator on $S \otimes E$ depends on the geometry of $E$. A condition for such converse to hold is now given. 
Proposition 4.6. Let $S$ be a Dirac bundle with positive Weitzenböck operator and let $E$ be any euclidean bundle. Let $\Theta$ be the bilinear form on $S \otimes E$ determined by

$$
\Theta(\sigma \otimes \xi, \tau \otimes \zeta)=\operatorname{tr}(\omega \mapsto\langle\omega \cdot \sigma, \tau\rangle\langle R(\omega) \xi, \zeta\rangle)
$$

The Weitzenböck operator on $S \otimes E$ is positive semi-definite if and only if

$$
\langle\mathcal{R} \sigma, \sigma\rangle\langle\mathcal{R} \tau, \tau\rangle|\xi|^{2}|\zeta|^{2}-\langle\mathcal{R} \sigma, \tau\rangle^{2}\langle\xi, \zeta\rangle^{2}-2\langle\mathcal{R} \sigma, \tau\rangle \Theta-\Theta^{2} \geq 0,
$$

for all $\sigma \otimes \xi, \tau \otimes \zeta \in S \otimes E$ over the same point. Furthermore, it is positive definite if the inequality is strict for nonzero vectors.

Proof. It follows from (4.3) and (4.4) that

$$
\langle\mathcal{R}(\sigma \otimes \xi), \tau \otimes \zeta\rangle=\langle\mathcal{R} \sigma, \tau\rangle\langle\xi, \zeta\rangle+\Theta(\sigma \otimes \xi, \tau \otimes \zeta)
$$

Notice that $\Theta(\eta, \eta)=0$ for any $\eta \in S \otimes E$ (cf. (4.3) in Lemma4.4). For linearly independent $\sigma \otimes \xi$ and $\tau \otimes \zeta$ in $S \otimes E$,

$$
\left[\begin{array}{cc}
\langle\mathcal{R} \sigma, \sigma\rangle|\xi|^{2} & \langle\mathcal{R} \sigma, \tau\rangle\langle\xi, \zeta\rangle+\Theta \\
\langle\mathcal{R} \sigma, \tau\rangle\langle\xi, \zeta\rangle+\Theta & \langle\mathcal{R} \tau, \tau\rangle|\zeta|^{2}
\end{array}\right]
$$

is the matrix associated to $\mathcal{R}$ restricted to the subspace generated by $\sigma \otimes \xi$ and $\tau \otimes \zeta$. Observe that the left-hand side of (4.5) is nothing but the determinant of this matrix; from which the conclusion follows.

An immediate consequence is the following result.

Proposition 4.7. Let $S$ be a Dirac bundle with positive semi-definite (resp. definite) Weitzenböck operator and let $E$ be a euclidean bundle with connection. If $\Theta \equiv 0$ then the Weitzenböck operator on $S \otimes E$ is positive semi-definite (resp. definite).

Proof. If $\Theta \equiv 0$, then all that is left to verify (4.5) is that

$$
\langle\mathcal{R} \sigma, \sigma\rangle\langle\mathcal{R} \tau, \tau\rangle|\xi|^{2}|\zeta|^{2} \geq\langle\mathcal{R} \sigma, \tau\rangle^{2}\langle\xi, \zeta\rangle^{2}
$$

Using Cauchy-Schwarz, it is sufficient to verify that

$$
\langle\mathcal{R} \sigma, \sigma\rangle\langle\mathcal{R} \tau, \tau\rangle \geq\langle\mathcal{R} \sigma, \tau\rangle^{2} .
$$

And this holds since $\mathcal{R}$ is positive semi-definite (resp. definite) on $S$.

Corollary 4.8. For $E$ flat, the Weitzenböck operator of $S \otimes E$ is positive semi-definite (resp. definite) if and only if the Weitzenböck operator of $S$ is positive semi-definite (resp. definite).

Proof. This is immediate from the fact that $\Theta$ vanishes identically in this case. 


\section{TWISTOR SECTIONS}

The purpose of this section is to prove Theorem B and Theorem C. To do this, we first introduce the twistor and Killing equations for sections of Dirac bundles and we establish some natural extensions of well-known facts in Spin Geometry.

Definition 5.1 (Twistor section). A section $\sigma$ of a Dirac bundle $S$ is a twistor section if it satisfies

$$
\nabla_{X} \sigma+\frac{1}{n} X \cdot D \sigma=0
$$

for all $X \in T M$.

Definition 5.2 (Killing section). A section $\sigma$ of a Dirac bundle $S$ is a Killing section with constant $\lambda$ if it satisfies

$$
\nabla_{X} \sigma=\lambda X \cdot \sigma
$$

for all $X \in T M$.

Equations (5.1) and (5.2) are respectively the twistor and Killing equations on $S$.

The following result is a natural extension to Dirac bundles of a result for spin bundles (see the proposition in [7] p. 117).

Proposition 5.3. Let $\sigma$ be a Killing section with constant $\lambda$ of a Dirac bundle $S$ over an $n$-dimensional $M$.

(i) If $M$ is connected, then $\sigma$ vanishes identically if it vanishes at a point.

(ii) The section $\sigma$ is also twistor. Moreover, it is an eigensection of the Dirac operator with eigenvalue $-n \lambda$.

Proof. The proof is straightforward and it is essentially identical to the proof of the proposition in [7] p. 117. The difference is that therein a hermitian inner product is used 3

In the case of spin bundles, the classical Bochner identity implies explicit conditions for the twistor equation to be satisfied. These formulas relate the Dirac operator and the curvature to a solution of the twistor equation. In the Dirac bundle case, the following result shows that there also exist explicit conditions linking the generalized Bochner identity (3.25) and the twistor equation (5.1). It is a generalization of Proposition 2 in [16] p. 335 and of the first two formulas of Theorem 3 in [4] p. 24. In analogy to the expressions found in the latter, we define the Ricci curvature operator $\operatorname{Ric}_{X}: S \rightarrow S$ of a Dirac bundle $S$ by

$$
\operatorname{Ric}_{X} \sigma=2 \sum_{i=1}^{n} e_{i} \cdot R\left(e_{i}, X\right) \sigma,
$$

where $e_{1}, \ldots, e_{n}$ is a local orthonormal frame.

\footnotetext{
${ }^{3} \mathrm{~A}$ result corresponding to the third part of that proposition is not included here since in the euclidean case $\left\langle e_{i} \cdot \sigma, \sigma\right\rangle=0$, and thus the vector field $\sum\left\langle e_{i} \cdot \sigma, \sigma\right\rangle e_{i}$ vanishes identically.
} 
Theorem 5.4. Let $S$ be a Dirac bundle over a compact $M$. A section $\sigma$ of $S$ is a twistor section if and only if

$$
D^{2} \sigma=\frac{n}{n-1} \mathcal{R} \sigma
$$

Moreover, in this case,

$$
\nabla_{X} D \sigma=\frac{n}{n-2}\left[\frac{1}{n-1} X \cdot \mathcal{R}-\frac{1}{2} \operatorname{Ric}_{X}\right] \sigma .
$$

Proof. Let $\sigma$ be a twistor section of $S$ and let $e_{1}, \ldots, e_{n}$ be a local orthonormal frame. Then from (3.22) and(5.1),

$$
\begin{aligned}
\nabla^{*} \nabla \sigma & =-\sum_{j=1}^{n} \nabla_{e_{j}, e_{j}}^{2} \sigma=-\sum_{j=1}^{n}\left(\nabla_{e_{j}} \nabla_{e_{j}} \sigma-\nabla_{\nabla_{e_{j}} e_{j}} \sigma\right) \\
& =\frac{1}{n} \sum_{j=1}^{n}\left(\nabla_{e_{j}}\left(e_{j} \cdot D \sigma\right)-\nabla_{e_{j}} e_{j} \cdot D \sigma\right)=\frac{1}{n} D^{2} \sigma .
\end{aligned}
$$

At this point (5.3) follows from the general Bochner identity (3.25).

Conversely, if $\sigma \in \Gamma(S)$ satisfies (5.3) then, again using (3.25), $\sigma$ satisfies

$$
\nabla^{*} \nabla \sigma=\frac{1}{n} D^{2} \sigma
$$

Using this, (3.14), (3.21) and (3.23), it follows that

$$
\begin{aligned}
\sum_{j=1}^{n}\left\|\nabla_{e_{j}} \sigma+\frac{1}{n} e_{j} \cdot D \sigma\right\|^{2} & =\sum_{j=1}^{n}\left\{\left\|\nabla_{e_{j}} \sigma\right\|^{2}+\frac{2}{n}\left(e_{j} \cdot D \sigma, \nabla_{e_{j}} \sigma\right)+\frac{1}{n}\|D \sigma\|^{2}\right\} \\
& =\left(\nabla^{*} \nabla \sigma, \sigma\right)-\frac{2}{n}(D \sigma, D \sigma)+\frac{1}{n}(D \sigma, D \sigma) \\
& =\left(\nabla^{*} \nabla \sigma, \sigma\right)-\frac{1}{n}\left(D^{2} \sigma, \sigma\right)=0 .
\end{aligned}
$$

So that $\nabla_{e_{j}} \sigma+\frac{1}{n} e_{j} \cdot D \sigma=0$ for $j=1, \ldots, n$; from which (5.1) follows.

To prove (5.4) let $\sigma$ be a twistor section, and $X, Y \in T M$ over the same point. Then,

$$
R(X, Y) \sigma=\frac{1}{n}\left(X \cdot \nabla_{Y} D \sigma-Y \cdot \nabla_{X} D \sigma\right) .
$$

Indeed, for two commuting vector fields $X, Y \in \mathfrak{X}(M)$,

$$
\begin{aligned}
R(X, Y) \sigma & =\nabla_{X} \nabla_{Y} \sigma-\nabla_{Y} \nabla_{X} \sigma \\
& =\frac{1}{n}\left(\nabla_{Y}(X \cdot D \sigma)-\nabla_{X}(Y \cdot D \sigma)\right) \\
& =\frac{1}{n}\left(X \cdot \nabla_{Y} D \sigma-Y \cdot \nabla_{X} D \sigma\right) .
\end{aligned}
$$


Since this is tensorial (5.5) holds. Lastly, using (5.3) and (5.5),

$$
\begin{aligned}
\frac{1}{2} \operatorname{Ric}_{X} \sigma & =\sum_{i=1}^{n} e_{i} \cdot R\left(e_{i}, X\right) \sigma \\
& =\frac{1}{n} \sum_{i=1}^{n}\left(e_{i} \cdot e_{i} \cdot \nabla_{X} D \sigma-e_{i} \cdot X \cdot \nabla_{e_{i}} D \sigma\right) \\
& =-\nabla_{X} D \sigma+\frac{1}{n} X \cdot D^{2} \sigma+\frac{2}{n} \sum_{i=1}^{n}\left\langle e_{i}, X\right\rangle \nabla_{e_{i}} D \sigma \\
& =-\nabla_{X} D \sigma+\frac{1}{n-1} X \cdot \mathcal{R} \sigma+\frac{2}{n} \nabla_{X} D \sigma \\
& =\frac{2-n}{n} \nabla_{X} D \sigma+\frac{1}{n-1} X \cdot \mathcal{R} \sigma
\end{aligned}
$$

from which (5.4) follows.

Consider for each $X \in \mathfrak{X}(M)$, the endomorphism $\mathcal{K}_{X}: S \rightarrow S$ given by

$$
\mathcal{K}_{X}=\frac{n}{n-2}\left[\frac{1}{n-1} L_{X} \circ \mathcal{R}-\frac{1}{2} \operatorname{Ric}_{X}\right],
$$

where $L_{X}: S \rightarrow S$ is the Clifford multiplication by $X$. By (5.1) and (5.4),

$$
\nabla_{X} \sigma=-\frac{1}{n} L_{X} \circ D \sigma \quad \nabla_{X} D \sigma=\mathcal{K}_{X} \sigma
$$

which suggests the following result (cf. [4] p. 26).

Theorem 5.5. On $E=S \oplus S$, with respect to the connection $\nabla^{E}: \Gamma(E) \rightarrow \Gamma\left(T^{*} M \otimes E\right)$ given by the matrix

$$
\left(\begin{array}{cc}
\nabla & \frac{1}{n} L \\
-\mathcal{K} & \nabla
\end{array}\right)
$$

a section $\sigma$ is a twistor section if and only if $(\sigma, D \sigma)$ is $\nabla^{E}$-parallel.

Proof. That for a twistor section $\sigma, \nabla^{E}(\sigma, D \sigma)=0$ is immediate from (5.7). Conversely, let $(\sigma, \tau)$ be $\nabla^{E}$-parallel. Then $\nabla_{X} \sigma=-\frac{1}{n} X \cdot \tau$ holds true for all $X \in T M$ and thus

$$
D \sigma=\sum_{i=1}^{n} e_{i} \cdot \nabla_{e_{i}} \sigma=\sum_{i=1}^{n} e_{i} \cdot\left(-\frac{1}{n} e_{i} \cdot \tau\right)=\tau,
$$

which establishes the claim.

Corollary 5.6. A twistor section $\sigma$ is determined by $\sigma_{p_{0}}$ and $D \sigma_{p_{0}}$ for a single $p_{0}$.

Corollary 5.7. The space of solutions to the twistor equation is finite-dimensional and its dimension is bounded by $2 \mathrm{rkS}$.

Corollary 5.8. If for a twistor section $\sigma$, both $\sigma$ and $D \sigma$ vanish simultaneously at a point, then $\sigma$ vanishes identically. 
The form of (5.2), as well as Theorem 5.5, suggest the use of modified connections (cf. [7] pp. 114-115) such as the one in the following technical result.

Lemma 5.9. Let $f: M \rightarrow \mathbb{R}$ be any smooth function and let $\nabla^{f}$ be the covariant derivative on $S$ given by

Then $\nabla^{f}$ is metric,

$$
\nabla_{X}^{f} \sigma=\nabla_{X} \sigma+f X \cdot \sigma
$$

$$
\nabla^{f *} \nabla^{f} \sigma=\nabla^{*} \nabla \sigma-\nabla f \cdot \sigma-2 f D \sigma+n f^{2} \sigma
$$

and

$$
(D-f)^{2} \sigma=\nabla^{f *} \nabla^{f} \sigma+\mathcal{R} \sigma+(1-n) f^{2} \sigma .
$$

Proof. To see that $\nabla^{f}$ is metric, notice that

$$
\left\langle\nabla_{X}^{f} \sigma, \tau\right\rangle+\left\langle\sigma, \nabla_{X}^{f} \tau\right\rangle=\left\langle\nabla_{X} \sigma, \tau\right\rangle+\left\langle\sigma, \nabla_{X} \tau\right\rangle
$$

Now, let $e_{1}, \ldots, e_{n}$ be a local orthonormal frame. Then

$$
\begin{aligned}
\nabla_{e_{j}}^{f} \nabla_{e_{j}}^{f} \sigma & =\nabla_{e_{j}} \nabla_{e_{j}} \sigma+\nabla_{e_{j}}\left(f e_{j} \cdot \sigma\right)+f e_{j} \cdot \nabla_{e_{j}} \sigma+f e_{j} \cdot\left(f e_{j} \sigma\right) \\
& =\nabla_{e_{j}} \nabla_{e_{j}} \sigma+e_{j}(f) e_{j} \cdot \sigma+f \nabla_{e_{j}} e_{j} \cdot \sigma+2 f e_{j} \cdot \nabla_{e_{j}} \sigma-f^{2} \sigma,
\end{aligned}
$$

and

$$
\nabla_{\nabla_{e_{j}} e_{j}}^{f} \sigma=\nabla_{\nabla_{e_{j}} e_{j}} \sigma+f \nabla_{e_{j}} e_{j} \cdot \sigma
$$

From these two expressions, it follows that

$$
\begin{aligned}
\nabla^{f *} \nabla^{f} \sigma & =-\sum_{j=1}^{n}\left[\nabla^{f}\right]_{e_{j}, e_{j}}^{2} \sigma \\
& =-\sum_{j=1}^{n}\left(\nabla_{e_{j}}^{f} \nabla_{e_{j}}^{f} \sigma-\nabla_{\nabla_{e_{j}} e_{j}}^{f} \sigma\right) \\
& =-\sum_{j=1}^{n}\left(\nabla_{e_{j}, e_{j}}^{2} \sigma+e_{j}(f) e_{j} \cdot \sigma+2 f e_{j} \cdot \nabla_{e_{j}} \sigma-f^{2} \sigma\right) \\
& =\nabla^{*} \nabla \sigma-\nabla f \cdot \sigma-2 f D \sigma+n f^{2} \sigma,
\end{aligned}
$$

which establishes (5.9) . To see (5.10), use (3.12) and the general Bochner identity,

$$
\begin{aligned}
(D-f)^{2} \sigma & =D^{2} \sigma-2 f D \sigma-\nabla f \cdot \sigma+f^{2} \sigma \\
& =\nabla^{*} \nabla \sigma+\mathcal{R} \sigma-2 f D \sigma-\nabla f \cdot \sigma+f^{2} \sigma \\
& =\nabla^{f *} \nabla^{f} \sigma+\mathcal{R} \sigma+(1-n) f^{2} \sigma .
\end{aligned}
$$

The following result characterizes Killing sections on Dirac bundles over compact manifolds.

Theorem 5.10. On a Dirac bundle $S$ over a compact $n$-dimensional $M$ the following are equivalent.

(i) A section $\sigma$ of $S$ is a Killing section with constant $\lambda$. 
(ii) A section $\sigma$ of $S$ satisfies $D \sigma=-n \lambda \sigma$ and $\mathcal{R} \sigma=\lambda^{2} n(n-1) \sigma$.

Proof. Let $\sigma$ be as in (i), then

$$
D \sigma=\sum_{i=1}^{n} e_{i} \cdot \nabla_{e_{i}} \sigma=\lambda \sum_{i=1}^{n} e_{i} \cdot e_{i} \cdot \sigma=-n \lambda \sigma .
$$

Thus $D^{2} \sigma=n^{2} \lambda^{2} \sigma$, which together with (5.3) establishes the remaining formula in (ii).

Conversely, let $\sigma$ be as in (ii) and use (5.10) with $f=-\lambda$ to obtain

$$
(D+\lambda)^{2} \sigma=\nabla^{-\lambda *} \nabla^{-\lambda} \sigma+\mathcal{R} \sigma+(1-n) \lambda^{2} \sigma .
$$

Using both conditions of (ii) in this last formula immediately yields that

$$
\nabla^{-\lambda *} \nabla^{-\lambda} \sigma=0
$$

Recall that the notion of connection laplacian can be defined on euclidean vector bundles and that properties such as (3.21) still hold. In particular, this is the case of $S$ with metric connection $\nabla^{-\lambda}$. Therefore, since $M$ is compact it follows that $\sigma$ is $\nabla^{-\lambda}$-parallel. This is exactly the Killing condition (5.2) for constant $\lambda$.

To end this section we provide another characterization of the Killing condition in terms of a lower bound for the square of the eigenvalues of the Dirac operator.

Proposition 5.11. Let $S$ be a Dirac bundle over a compact $M$ and let $\mu$ be an eigenvalue of $D$, then

$$
\mu^{2} \geq \frac{n}{n-1} R_{0}
$$

where

$$
R_{0}=\min _{|\sigma|=1}\langle\mathcal{R} \sigma, \sigma\rangle .
$$

Moreover, the equality holds if and only if $\sigma$ is a Killing section with constant $\mp \sqrt{\frac{R_{0}}{n(n-1)}}$ and $\mathcal{R}$ is positive semi-definite.

Proof. Let $\sigma$ be an eigensection of $D$ with eigenvalue $\mu$. On (5.10) let $f=\frac{\mu}{n}$ to obtain

$$
\mu^{2} \frac{n-1}{n} \sigma=\nabla^{\frac{\mu}{n}} * \nabla^{\frac{\mu}{n}} \sigma+\mathcal{R} \sigma .
$$

Since $M$ is compact, (3.21) yields

$$
\mu^{2} \frac{n-1}{n}(\sigma, \sigma)=\left(\nabla^{\frac{\mu}{n}} \sigma, \nabla^{\frac{\mu}{n}} \sigma\right)+(\mathcal{R} \sigma, \sigma) \geq R_{0}(\sigma, \sigma)
$$

which implies (5.11).

Furthermore, if equality holds, then $\sigma$ is $\nabla^{\frac{\mu}{n}}$-parallel; i.e. $\sigma$ is Killing with constant $\lambda=-\frac{\mu}{n}=\mp \sqrt{\frac{R_{0}}{n(n-1)}}$. Conversely, if $\sigma$ is a Killing section with constant $\lambda=\mp \sqrt{\frac{R_{0}}{n(n-1)}}$, then by Theorem 5.10 ,

$$
D \sigma=-n \lambda \sigma= \pm \sqrt{\frac{n}{n-1} R_{0}} \sigma
$$


Hence, $\mu= \pm \sqrt{\frac{n}{n-1} R_{0}}$ is an eigenvalue of $D$ and equality in (5.11) holds.

\section{The Clifford Bundle Case}

The main purpose of this section is to prove Theorem D. First, we study the interplay of the Weitzenböck operator with the additional structure of the Clifford bundle. Then, in Proposition 6.2 we prove that the trace of the Weitzenböck operator when restricted to $p$-forms is a multiple of the scalar curvature. Lastly, we restrict our attention to spaces of constant sectional curvature and obtain explicit formulas for the Weitzenböck operator.

Under the standard identification with the exterior bundle, the Dirac operator is given as $D=d+d^{*}$, from which

$$
D^{2}=\Delta
$$

where $\Delta$ is the usual Hodge laplacian (see [15] p. 123). A simple computation shows that for any function $f$ and any $n$-form $\omega$

$$
\mathcal{R}(f)=\mathcal{R}(\omega)=0 .
$$

Furthermore, for any 1-form $\varphi$,

$$
\mathcal{R} \varphi=\operatorname{Ric}(\varphi)
$$

and $\mathcal{R}$ is positive semi-definite (resp. definite) whenever the curvature operator $R$ : $\Lambda^{2}(M) \rightarrow \Lambda^{2}(M)$ is positive semi-definite (resp. definite) (see [15] pp. 156-160).

Following the standard multi-index notation for forms (cf. [12]), for any $e_{1}, \ldots, e_{n}$ local orthonormal frame and for any ordered $I \subset\{1, \ldots, n\}$ denote by $e_{I}$ the corresponding exterior form and denote by $I^{\prime}=\{1, \ldots, n\} \backslash I$, its ordered complement. Since the Hodge operator $*$ is given by the equation

$$
\varphi \wedge * \psi=\langle\varphi, \psi\rangle * 1
$$

where $* 1=e_{\{1, \ldots, n\}}$, it follows $* e_{I}=(-1)^{\ell_{I}} e_{I^{\prime}}$, for an appropriate $\ell_{I}$.

Proposition 6.1. On the Clifford bundle the Weitzenböck operator and the Hodge operator commute.

Proof. In view of (3.25), it is enough to prove that $*$ commutes with $D^{2}$ and $\nabla^{*} \nabla$. Since the operator $D^{2}$ satisfies (6.1), it commutes with $*$. Hence, by (3.22), it is enough to prove that $\nabla$ commutes with $*$. To see this, notice that

$$
* 1 \cdot \varphi=(-1)^{p(n-p)+\frac{p(p+1)}{2}} * \varphi
$$

for any $p$-form $\varphi$ (see (5.35) in [15] p. 129). Now, since $* 1$ is parallel,

$$
\begin{aligned}
\nabla_{X}(* \varphi) & =(-1)^{p(n-p)+\frac{p(p+1)}{2}} \nabla_{X}(* 1 \cdot \varphi) \\
& =(-1)^{p(n-p)+\frac{p(p+1)}{2}} * 1 \cdot \nabla_{X} \varphi \\
& =* \nabla_{X} \varphi
\end{aligned}
$$

where (6.5) is used for $\varphi$ and $\nabla_{X} \varphi$. 
Proposition 6.2. On the exterior bundle of $M$, for any $p=1, \ldots, n-1$, the trace of the restriction $\mathcal{R}_{p}$ of the Weitzenböck operator to p-forms is

$$
\operatorname{tr} \mathcal{R}_{p}=\left(\begin{array}{c}
n-2 \\
p-1
\end{array}\right) s
$$

where $s$ is the scalar curvature of $M$. In particular, in light of (6.2), the trace of the total Weitzenböck operator is

$$
\operatorname{tr} \mathcal{R}=2^{n-2} s
$$

Proof. As is well known, the curvature operator on 2-forms is determined by

$$
\left\langle\mathbf{R}\left(e_{i} \wedge e_{j}\right), e_{k} \wedge e_{\ell}\right\rangle=\left\langle R\left(e_{i}, e_{j}\right) e_{\ell}, e_{k}\right\rangle,
$$

and it is self-adjoint. In terms of this operator,

$$
\langle\mathcal{R} \varphi, \varphi\rangle=\frac{1}{4} \sum_{\xi, \zeta \in B}\langle\mathbf{R} \xi, \zeta\rangle\langle[\xi, \varphi],[\zeta, \varphi]\rangle,
$$

for any orthonormal basis $B$ of the space of 2-forms (cf. [15] p. 159). Using the above formula, the trace of $\mathcal{R}_{p}$ is given by

$$
\operatorname{tr} \mathcal{R}_{p}=\sum_{|I|=p}\left\langle\mathcal{R} e_{I}, e_{I}\right\rangle=\frac{1}{4} \sum_{|I|=p} \sum_{\xi \in B} \lambda_{\xi}\left|\left[\xi, e_{I}\right]\right|^{2},
$$

assuming $B$ is a basis of eigen-2-forms of $\mathbf{R}$, with $\lambda_{\xi}$ the corresponding eigenvalue for $\xi \in B$. To compute the right-hand side of (6.8), let $\xi=\frac{1}{2} \sum \xi^{i j} e_{i} e_{j}$ to yield that

$$
\left|\left[\xi, e_{I}\right]\right|^{2}=\frac{1}{4} \sum_{i, j, k, \ell} \xi^{i j} \xi^{k \ell}\left\langle\left[e_{i} e_{j}, e_{I}\right],\left[e_{k} e_{\ell}, e_{I}\right]\right\rangle .
$$

An easy computation gives that

$$
\left[e_{i} e_{j}, e_{I}\right]= \begin{cases}0 & \text { if }\{i, j\} \subset I \\ 0 & \text { if }\{i, j\} \subset I^{\prime} \\ 2 e_{i} e_{j} e_{I} & \text { otherwise }\end{cases}
$$

from which it follows that $\left\langle\left[e_{i} e_{j}, e_{I}\right],\left[e_{k} e_{\ell}, e_{I}\right]\right\rangle$ is nonzero only when $\{i, j\}=\{k, \ell\}$ provided that $\{i, j\}$ is not contained in either of $I$ or $I^{\prime}$. Taking into account these four cases, (6.9) and (6.10) imply that

$$
\begin{aligned}
\left|\left[\xi, e_{I}\right]\right|^{2} & =\sum_{i \in I} \sum_{j \in I^{\prime}}\left(\xi^{i j}\right)^{2}-\sum_{i \in I} \sum_{j \in I^{\prime}} \xi^{i j} \xi^{j i}-\sum_{i \in I^{\prime}} \sum_{j \in I} \xi^{i j} \xi^{j i}+\sum_{i \in I^{\prime}} \sum_{j \in I}\left(\xi^{i j}\right)^{2} \\
& =4 \sum_{i \in I} \sum_{j \in I^{\prime}}\left(\xi^{i j}\right)^{2}
\end{aligned}
$$


since $\xi^{j i}=-\xi^{i j}$. Replace $\left|\left[\xi, e_{I}\right]\right|^{2}$ by this last expression in (6.8) to obtain

$$
\operatorname{tr} \mathcal{R}_{p}=\sum_{\xi \in B} \lambda_{\xi} \sum_{|I|=p} \sum_{i \in I} \sum_{j \in I^{\prime}}\left(\xi^{i j}\right)^{2}
$$

Observe that for fixed $i$ and $j$, there are $\left(\begin{array}{l}n-2 \\ p-1\end{array}\right)$ subsets $I$ for which $i \in I$ and $j \in I^{\prime}$. This implies that

$$
\sum_{|I|=p} \sum_{i \in I} \sum_{j \in I^{\prime}}\left(\xi^{i j}\right)^{2}=\left(\begin{array}{c}
n-2 \\
p-1
\end{array}\right) \sum_{i, j}\left(\xi^{i j}\right)^{2}=2\left(\begin{array}{c}
n-2 \\
p-1
\end{array}\right),
$$

since $\xi \in B$ has norm 1 . Finally, since

$$
\sum_{\xi \in B} \lambda_{\xi}=\operatorname{tr} \mathbf{R}=\frac{s}{2}
$$

use (6.11) and (6.12) to yield (6.6). A standard computation yields (6.7).

Theorem 5.4 can be used to solve the twistor equation completely on $\mathrm{C} \ell\left(S^{n}\right)$. In order to do that the following preliminary result is needed, which might be of independent interest.

Lemma 6.3. Let $M$ have constant sectional curvature $\kappa$. On the exterior bundle, the restriction $\mathcal{R}_{p}$ of the Weitzenböck operator to $p$-forms is

$$
\mathcal{R}_{p} \varphi=\kappa p(n-p) \varphi
$$

Proof. Let $e_{1}, \ldots, e_{n}$ be a local orthonormal frame. From [15] p. 111, the curvature operator on $\mathrm{C} \ell(M)$ can be written as

$$
R(X, Y) \varphi=\frac{1}{2} \sum_{k<l}\left\langle R(X, Y) e_{k}, e_{l}\right\rangle\left[e_{k} \cdot e_{l}, \varphi\right]
$$

In particular, it follows from this formula and (6.10) that

$$
\begin{aligned}
R\left(e_{i}, e_{j}\right) e_{I} & =\frac{1}{4} \sum_{k, l}\left\langle R\left(e_{i}, e_{j}\right) e_{k}, e_{l}\right\rangle\left[e_{k} \cdot e_{l}, \varphi\right] \equiv \frac{1}{4} \sum_{k, \ell} R_{i j k \ell}\left[e_{k} \cdot e_{l}, \varphi\right] \\
& =\frac{1}{2} \sum_{k \in I} \sum_{\ell \in I^{\prime}} R_{i j k \ell} e_{k} \cdot e_{\ell} \cdot e_{I}+\frac{1}{2} \sum_{k \in I^{\prime}} \sum_{\ell \in I} R_{i j k \ell} e_{k} \cdot e_{\ell} \cdot e_{I} \\
& =\sum_{k \in I} \sum_{\ell \in I^{\prime}} R_{i j k \ell} e_{k} \cdot e_{\ell} \cdot e_{I} .
\end{aligned}
$$

Since $\kappa$ is constant, $R(X, Y) Z=\kappa(\langle Y, Z\rangle X-\langle X, Z\rangle Y)$, and thus

$$
\begin{aligned}
\mathcal{R}_{p} e_{I} & =\frac{1}{2} \sum_{i, j} \sum_{k \in I} \sum_{\ell \in I^{\prime}} \kappa\left(\delta_{i \ell} \delta_{j k}-\delta_{i k} \delta_{j \ell}\right) e_{i} \cdot e_{j} \cdot e_{k} \cdot e_{\ell} \cdot e_{I} \\
& =\sum_{k \in I} \sum_{\ell \in I^{\prime}} \kappa e_{I}=\kappa p(n-p) e_{I} .
\end{aligned}
$$


Theorem 6.4. The twistor sections of $\mathrm{C} \ell\left(S^{n}\right), n \geq 2$, are given by

$$
\sigma=c_{1}+d f_{1}+d^{*}\left(* f_{2}\right)+* c_{2},
$$

with constants $c_{1}, c_{2}$ and functions $f_{1}, f_{2}: S^{n} \rightarrow \mathbb{R}$ such that $\Delta f_{i}=n f_{i}$.

Solutions to $\Delta f=n f$ are well known to exist (see [5] for details).

Proof of Theorem 6.4. From Theorem 5.4 to find twistor sections is equivalent to solving (5.3). Let $\sigma=\sigma_{0}+\cdots+\sigma_{n}$, where $\sigma_{p}$ is of degree $p$. From (6.1) and Lemma 6.3, $\sigma$ is a twistor section if and only if

$$
\Delta \sigma_{p}=\frac{n}{n-1} p(n-p) \sigma_{p}, \quad p=0,1, \ldots, n .
$$

Hereinafter the fact that $\Delta$ commutes with $d$ and $d^{*}$ is used extensively; e.g. if $\sigma_{0}=g$ and $\sigma_{n}=* h$, by (6.16) $g$ and $h$ are harmonic and thus constant.

Now, for $p \neq 0$ and $p \neq n$, the smallest positive eigenvalue $\mu_{n, p}$ for $\Delta$ on the space of $p$-forms of $S^{n}$ is given as the minimum

$$
\mu_{n, p}=\min \{p(n-p+1),(p+1)(n-p)\},
$$

where $p(n-p+1)$ (resp. $(p+1)(n-p))$ corresponds to the first positive eigenvalue when restricted to closed (resp. co-closed) $p$-forms 4

For $p=1$, (6.16) is written as $\Delta \sigma_{1}=n \sigma_{1}$ and thus

$$
\sigma_{1}=d f_{1}+d^{*} \eta
$$

with $f_{1}, \eta \in \operatorname{ker}(\Delta-n)$. The case $n=2$ follows since $\eta=* f_{2}$. For $n=3$ and $p=1$, since $p(n-p+1)=3,(p+1)(n-p)=4$ and (6.16) is $\Delta \sigma_{1}=3 \sigma_{1}$, it follows that any solution $\sigma_{1}$ must be closed and thus in (6.18) $d^{*} \eta=0$. For $n=3$ and $p=2$ the analysis is analogous. Therefore, there exist functions $f_{1}$ and $f_{2}$ in the kernel of $\Delta-n$ such that

$$
\sigma_{1}=d f_{1} \quad \text { and } \quad \sigma_{n-1}=d^{*}\left(* f_{2}\right),
$$

which proves the claim for $n=3$. For $n \geq 4$, notice that for $p=2, \ldots, n-2$,

$$
\frac{n}{n-1} p(n-p)<\mu_{n, p},
$$

and thus the only solution $\sigma_{p}$ to (6.16) is the trivial solution; therefore

$$
\sigma=\sigma_{0}+\sigma_{1}+\sigma_{n-1}+\sigma_{n} .
$$

Since $n<\mu_{n, 2}$, for $p=1$ it follows that in (6.18) $\eta \equiv 0$. The case $p=n-1$ is analogous. Therefore, there exist functions $f_{1}$ and $f_{2}$ as in (6.19), which finishes the proof.

\footnotetext{
${ }^{4}$ These formulas are due to Calabi (unpublished). See [9] for details.
} 


\section{A. The two-Dimensional Case}

Here we analyze the Killing equation on Clifford bundles over surfaces. This equation is much more restrictive than the twistor equation as the following result shows.

Theorem A.1. For a compact 2-dimensional $M$, there are nontrivial Killing sections of $\mathrm{C} \ell(M)$ with value $\lambda$ if and only if $\lambda=0$ and the gaussian curvature $\kappa \equiv 0$. Furthermore any such section is parallel.

To prove this, a couple of preliminary observations are required. Firstly, using the general fact that $\mathrm{C} \ell(M)$ splits into a Whitney sum of its even and odd parts, it is easy to see that the Clifford multiplication and the Dirac operator map the even into the odd and viceversa, while the Weitzenböck operator preserves them.

By (6.2) and (6.3), it follows that for a two-dimensional $M$,

$$
\mathcal{R} \sigma=\kappa \sigma_{1},
$$

for any section $\sigma \in \mathrm{C} \ell(M)$, where $\sigma_{1}$ is the odd part of $\sigma$.

Lemma A.2. For a compact 2-dimensional $M$, let $\sigma$ an eigensection of $D$ with eigenvalue $\lambda \neq 0$. Then the even and odd parts of $\sigma$ are non trivial and

$$
\lambda^{2} \geq \min \kappa \text {. }
$$

Proof. Let $\sigma_{0}$ and $\sigma_{1}$ be the even and odd parts of $\sigma$, respectively. Since $D$ shifts degree, $D \sigma_{0}=\lambda \sigma_{1}$ and $D \sigma_{1}=\lambda \sigma_{0}$. In particular, since $\lambda \neq 0$ and $\sigma$ is nontrivial then so are $\sigma_{0}$ and $\sigma_{1}$. Finally, using the Bochner identity and (A.1),

$$
\lambda^{2}\left\|\sigma_{1}\right\|^{2}=\left\|\nabla \sigma_{1}\right\|^{2}+\kappa\left\|\sigma_{1}\right\|^{2} \geq \kappa\left\|\sigma_{1}\right\|^{2},
$$

from which (A.2) follows.

The estimate from (A.2) is slightly better than that of (5.11), albeit in this trivial case.

Proof of Theorem A.1. Since $\sigma$ is a nontrivial Killing section, Theorem 5.10 implies that

$$
\mathcal{R} \sigma=2 \lambda^{2} \sigma \quad \text { and } \quad D \sigma=-2 \lambda \sigma .
$$

In light of (6.3), the first equation is $\kappa \sigma_{1}=2 \lambda^{2} \sigma$, which is satisfied if and only if $2 \lambda^{2} \sigma_{0}=0$ and $\kappa=2 \lambda^{2}$. This, together with the second equation in (A.3) and Lemma A.2 implies that $\lambda=0$ and $\kappa \equiv 0$. Conversely, $\lambda=0$ means that $\sigma$ is parallel; $\kappa \equiv 0$ that $M$ is flat, which guarantees the existence of nontrivial parallel sections.

Acknowledgements. The first two authors are supported by the Cátedras Conacyt Program Project No. 61. The third author was supported by the FORDECYT Grant No. 265667 (CONACYT) as a postdoctoral fellow. The third author wishes to express his gratitude to the Instituto de Matemáticas Oaxaca (UNAM) for its hospitality, where most of this project was developed. 


\section{REFERENCES}

[1] M. F. Atiyah and R. S. Ward. Instantons and algebraic geometry. Comm. Math. Phys., 55(2):117-124, 1977. ISSN 0010-3616. URL http://projecteuclid.org/euclid.cmp/1103900980.

[2] M. F. Atiyah, N. J. Hitchin, and I. M. Singer. Self-duality in fourdimensional Riemannian geometry. Proc. Roy. Soc. London Ser. A, 362 (1711):425-461, 1978. ISSN 0962-8444. doi: 10.1098/rspa.1978.0143. URL https://doi.org/10.1098/rspa.1978.0143.

[3] Christian Bär. The Dirac operator on space forms of positive curvature. J. Math. Soc. Japan, 48(1):69-83, 1996. ISSN 0025-5645. doi: 10.2969/jmsj/04810069. URL https://doi.org/10.2969/jmsj/04810069.

[4] Helga Baum, Thomas Friedrich, Ralf Grunewald, and Ines Kath. Twistors and Killing spinors on Riemannian manifolds, volume 124 of Teubner-Texte zur Mathematik [Teubner Texts in Mathematics]. B. G. Teubner Verlagsgesellschaft mbH, Stuttgart, 1991. ISBN 3-8154-2014-8. With German, French and Russian summaries.

[5] Marcel Berger. A panoramic view of Riemannian geometry. Springer-Verlag, Berlin, 2003. ISBN 3-540-65317-1. doi: 10.1007/978-3-642-18245-7. URL https://doi.org/10.1007/978-3-642-18245-7.

[6] Thomas Friedrich. On the conformal relation between twistors and Killing spinors. In Proceedings of the Winter School on Geometry and Physics (Srní, 1989), number 22, pages $59-75,1990$.

[7] Thomas Friedrich. Dirac operators in Riemannian geometry, volume 25 of Graduate Studies in Mathematics. American Mathematical Society, Providence, RI, 2000. ISBN 0-8218-2055-9. doi: 10.1090/gsm/025. URL https://doi.org/10.1090/gsm/025. Translated from the 1997 German original by Andreas Nestke.

[8] Thomas Friedrich and Olga Pokorná. Twistor spinors and solutions of the equation (E) on Riemannian manifolds. In Proceedings of the Winter School on Geometry and Physics (Srní, 1990), number 26, pages 149-154, 1991.

[9] Pierre Guerini and Alessandro Savo. Eigenvalue and gap estimates for the Laplacian acting on $p$-forms. Trans. Amer. Math. Soc., 356(1):319344, 2004. ISSN 0002-9947. doi: 10.1090/S0002-9947-03-03336-1. URL https://doi.org/10.1090/S0002-9947-03-03336-1.

[10] Katharina Habermann. The twistor equation on Riemannian manifolds. J. Geom. Phys., 7(4):469-488 (1991), 1990. ISSN 0393-0440. doi: 10.1016/0393-0440(90) 90002-K. URL https://doi.org/10.1016/0393-0440(90)90002-K.

[11] Katharina Habermann. Twistor spinors and their zeroes. J. Geom. Phys., 14 (1):1-24, 1994. ISSN 0393-0440. doi: 10.1016/0393-0440(94)90051-5. URL https://doi.org/10.1016/0393-0440(94)90051-5.

[12] Shoshichi Kobayashi. Differential geometry of complex vector bundles, volume 15 of Publications of the Mathematical Society of Japan. Princeton University Press, Princeton, NJ; Princeton University Press, Princeton, NJ, 1987. ISBN 0-691-08467X. doi: 10.1515/9781400858682. URL https://doi.org/10.1515/9781400858682. 
Kanô Memorial Lectures, 5.

[13] Shoshichi Kobayashi and Katsumi Nomizu. Foundations of differential geometry. Vol. I. Wiley Classics Library. John Wiley \& Sons, Inc., New York, 1996. ISBN 0-47115733-3. Reprint of the 1963 original, A Wiley-Interscience Publication.

[14] Wolfgang Kühnel and Hans-Bert Rademacher. Twistor spinors with zeros. Internat. J. Math., 5(6):877-895, 1994. ISSN 0129-167X. doi: 10.1142/S0129167X94000450. URL https://doi .org/10.1142/S0129167X94000450.

[15] H. Blaine Lawson, Jr. and Marie-Louise Michelsohn. Spin geometry, volume 38 of Princeton Mathematical Series. Princeton University Press, Princeton, NJ, 1989. ISBN 0-691-08542-0.

[16] André Lichnerowicz. Spin manifolds, Killing spinors and universality of the Hijazi inequality. Lett. Math. Phys., 13(4):331-344, 1987. ISSN 0377-9017. doi: 10.1007/ BF00401162. URL https://doi.org/10.1007/BF00401162.

[17] André Lichnerowicz. Killing spinors, twistor-spinors and Hijazi inequality. J. Geom. Phys., 5(1):1-18, 1988. ISSN 0393-0440. doi: 10.1016/0393-0440(88)90011-3. URL https://doi.org/10.1016/0393-0440(88)90011-3.

[18] R. Penrose. Twistor algebra. J. Mathematical Phys., 8:345-366, 1967. ISSN 0022-2488. doi: 10.1063/1.1705200. URL https://doi.org/10.1063/1.1705200.

[19] R Penrose. Twistor theory, its aims and achievements. In R. Penrose, D. W. Sciama, and C. J. Isham, editors, Quantum gravity, pages 268-407. Clarendon Press, Oxford, 1975. ISBN 0-19-851943-5.

[20] Roger Penrose and Wolfgang Rindler. Spinors and space-time. Vol. 2. Cambridge Monographs on Mathematical Physics. Cambridge University Press, Cambridge, second edition, 1988. ISBN 0-521-34786-6. Spinor and twistor methods in space-time geometry.

(†) Instituto de Matemáticas, Universidad Nacional Autónoma de México, Oaxaca de JuáREZ, MÉxiCO.

E-mail address: sholguin@im.unam.mx

(*) Instituto de Matemáticas, Universidad Nacional Autónoma de México, Oaxaca de JuáREZ, MÉXICO.

E-mail address: pedro.solorzano@matem.unam.mx

( $\ddagger$ Facultad de Economía. Universidad Autónoma de San Luis Potosí, San Luis Potosí, MÉxico.

E-mail address: ivan.tellez@uaslp.mx 